PROCEEDINGS OF THE

AMERICAN MATHEMATICAL SOCIETY

Volume 134, Number 11, November 2006, Pages 3171-3179

S 0002-9939(06)08308-0

Article electronically published on June 5, 2006

\title{
TOPOLOGICAL MIXING AND HYPERCYCLICITY CRITERION FOR SEQUENCES OF OPERATORS
}

\author{
JENG-CHUNG CHEN AND SEN-YEN SHAW
}

(Communicated by N. Tomczak-Jaegermann)

\begin{abstract}
For a sequence $\left\{T_{n}\right\}$ of continuous linear operators on a separable Fréchet space $X$, we discuss necessary conditions and sufficient conditions for $\left\{T_{n}\right\}$ to be topologically mixing, and the relations between topological mixing and the Hypercyclicity Criterion. Among them are: 1) topological mixing is equivalent to being hereditarily densely hypercyclic; 2) the Hypercyclicity Criterion with respect to the full sequence $\mathbb{N}$ implies topological mixing; 3) topological mixing implies the Hypercyclicity Criterion with respect to some sequence $\left\{n_{k}\right\} \subset \mathbb{N}$ that cannot be syndetic in general, and also implies condition (b) of the Hypercyclicity Criterion with respect to the full sequence. Applications to two examples of operators on the Fréchet space $H(\mathbb{C})$ of entire functions are also discussed.
\end{abstract}

\section{INTRODUCTION}

Let $X$ be a separable Fréchet space and denote by $L(X)$ the space of all continuous linear operators from $X$ to $X$. An operator $T \in L(X)$ is called hypercyclic if there exists an $x \in X$ (called a hypercyclic vector) such that its orbit $\operatorname{Orb}(T, x):=\left\{T^{n} x\right\}_{n \in \mathbb{N}}$ is dense in $X$. This property is an infinite-dimensional phenomenon, i.e., no finite-dimensional space admits a hypercyclic operator [19].

In 1929, Birkhoff [1] showed that the translation operator $T_{a}: f(z) \rightarrow f(z+a)$ $(a \neq 0)$ is hypercyclic on the Fréchet space $H(\mathbb{C})$ of entire functions. G. MacLane 21] proved that the differential operator $D: f \rightarrow f^{\prime}$ is also hypercyclic on $H(\mathbb{C})$. See [15, 3] for generalizations of these results. Recently, hypercyclic operators on arbitrary separable Fréchet spaces have been studied in [1]-3], 6]-10, 12]-19], and 22, 23. Hypercyclicity has also been considered for nonlinear mappings on topological spaces (see 44).

Being hypercyclic for $T$ is equivalent (see [18]) to a property called topological transitivity, which means that for any pair $U, V$ of nonempty open subsets of $X$, there exists some $n \in \mathbb{N}$ such that $T^{n}(U) \cap V \neq \emptyset$. Besides this equivalent condition, there are the following two sufficient conditions for hypercyclicity.

Received by the editors November 1, 2004 and, in revised form, December 18, 2004, March 29, 2005 and April 25, 2005.

2000 Mathematics Subject Classification. Primary 47A16, 47B37; Secondary 46A16, 47B33.

Key words and phrases. Hypercyclic sequence of operators, densely hypercyclic, topologically transitive, hereditarily densely hypercyclic, Hypercyclicity Criterion, topologically mixing.

This research was partially supported by the National Science Council of Taiwan. 
Hypercyclicity Criterion (cf. Kitai [19] and Gethner and Shapiro 14]). There exist an increasing sequence $\left\{n_{k}\right\} \subset \mathbb{N}$, two dense subsets $X_{0}, Y_{0}$, and mappings $S_{n_{k}}: Y_{0} \rightarrow X$ such that $T^{n_{k}} x \rightarrow 0$ for all $x \in X_{0}, S_{n_{k}} y \rightarrow 0$ for all $y \in Y_{0}$, and $T^{n_{k}} S_{n_{k}} y \rightarrow y$ for all $y \in Y_{0}$ (cf. [10, Definition 1.2]).

Bés and Peris [10] have shown two properties equivalent to the Hypercyclicity Criterion. One is the existence of a hereditarily hypercyclic subsequence, i.e., there exists a subsequence $\left\{n_{k}\right\}$ such that every subsequence of $\left\{T^{n_{k}}\right\}$ is hypercyclic. The other is weakly mixing, i.e., $T \oplus T$ is hypercyclic (or equivalently, $T \oplus T$ is topologically transitive). A not necessarily linear mapping $F$ is weakly mixing if and only if $F^{2}$ is hypercyclic and $F$ is flip transitive, which means that for any nonempty open sets $U, V$ there exists $n \in \mathbb{N}$ such that $F^{n}(U) \cap V \neq \emptyset$ and $F^{n}(V) \cap U \neq \emptyset$ (see [4]). Since any power $T^{n}$ of a hypercyclic linear operator $T$ is also hypercyclic (see [1]), the weaker condition "flip transitive" is surprisingly equivalent to "weakly mixing" for the case of linear operators. It is known (cf. [4]) that for a number $\theta$ such that $\frac{\theta}{\pi}$ is irrational, the rotation $R_{\theta}: S^{1} \rightarrow S^{1}$, defined by $R_{\theta}\left(e^{t}\right)=$ $e^{t+\theta}$, is hypercyclic but not weakly mixing. However, it is still unknown whether there is a linear operator which is hypercyclic but not weakly mixing (equivalently, does not satisfy the Hypercyclicity Criterion). The Hypercyclicity Criterion is also equivalent (see [23, Theorem 2.3]) to syndetical hypercyclicity, which means that for any syndetic sequence $\left\{n_{k}\right\}$, i.e., satisfying $\sup _{k}\left\{n_{k+1}-n_{k}\right\}<\infty$, the sequence $\left\{T^{n_{k}}\right\}$ is hypercyclic. Recently, Bermúdez et al. [5, Remark 3.3] showed that $\left\{T^{n}\right\}$ (or simply $T$ ) satisfies the Hypercyclicity Criterion with respect to the full sequence if and only if it satisfies the Hypercyclicity Criterion with respect to some syndetic sequence $\left\{n_{k}\right\}$.

Topological mixing (cf. [4, [13]). For any nonempty open sets $U, V$ there is some $N \in \mathbb{N}$ such that $T^{n}(U) \cap V \neq \emptyset$ for all $n \geq N$.

A theorem of Costakis and Sambarino [13. Theorem 1.1] states that if $\left\{T^{n}\right\}$ satisfies the Hypercyclicity Criterion with respect to some syndetic sequence $\left\{n_{k}\right\}$, then $T$ is topologically mixing.

In [8, 15, 18, 7, 20], the above hypercyclicity-related notions have been considered for sequences of (linear) operators. The purpose of this work is to discuss topological mixing and the Hypercyclicity Criterion for sequences of operators. In particular, necessary conditions and sufficient conditions for a sequence $\left\{T_{n}\right\}$ of operators to be topologically mixing, and the relations between topological mixing and the Hypercyclicity Criterion will be observed.

In Section 2, we first review related definitions and some known results (Theorems 2.2 and 2.3). Then we give some necessary conditions for hypercyclicity, hereditary hypercyclicity, and topological mixing (Proposition 2.5). Theorem 2.6 characterizes topological mixing by hereditarily dense hypercyclicity. Theorem 2.7 asserts that the Hypercyclicity Criterion with respect to the full sequence $\{n\}$ is sufficient for topological mixing, and every topologically mixing sequence of operators satisfies the Hypercyclicity Criterion for some sequence $\left\{n_{k}\right\} \subset \mathbb{N}$ and also satisfies (b) of the Hypercyclicity Criterion (see Definition 2.1) with respect to the full sequence. Specialization of the results (Theorems 2.6 and 2.7) to two examples of sequences of operators on $H(\mathbb{C})$ will be given in Section 3.

Finally, we remark that a statement which holds for the sequence $\left\{T^{n}\right\}$ is not necessarily true for a general sequence $\left\{T_{n}\right\}$. For instance, unlike in the case of a 
single operator (cf. 223, Theorem 2.3] and [13, Theorem 1.1]), for the sequence $\left\{T_{n}\right\}$, the Hypercyclicity Criterion is in general not equivalent to syndetical hypercyclicity (see Remark 2.4), and the Hypercyclicity Criterion with respect to some syndetic sequence in general does not imply topological mixing (see Remark 2.9(1)). On the other hand, neither does topological mixing imply the Hypercyclicity Criterion with respect to any syndetic sequence even for the sequence $\left\{T^{n}\right\}$ (see Remark 2.9(2)). This answers negatively a question raised by Costakis and Sambarino [13, p. 386].

\section{OBSERVATIONS ON TOPOLOGICAL MiXING AND HYPERCYCLICITY CRITERION}

We begin with the following definition.

Definition 2.1. Let $\left\{T_{n}\right\}$ be a sequence in $L(X)$.

(1) $\left\{T_{n}\right\}$ is hypercyclic (cf. [15]) if there exists a vector $x \in X$ (called hypercyclic vector) such that $\left\{T_{n} x ; n \in \mathbb{N}\right\}$ is dense in $X$. The set of all hypercyclic vectors of $\left\{T_{n}\right\}$ is denoted by $H C\left(\left\{T_{n}\right\}\right)$. When $H C\left(\left\{T_{n}\right\}\right)$ is a dense set, $\left\{T_{n}\right\}$ is called densely hypercyclic.

(2) $\left\{T_{n}\right\}$ is hereditarily (densely) hypercyclic (cf. [18 and [7]) if every subsequence of $\left\{T_{n}\right\}$ is (densely) hypercyclic.

(3) $\left\{T_{n}\right\}$ is said to be topologically transitive if for any given nonempty open sets $U, V$ there exists $n \in \mathbb{N}$ such that $T_{n}(U) \cap V \neq \emptyset$.

(4) $\left\{T_{n}\right\}$ is said to be topologically mixing if for any given nonempty open sets $U, V$ there exists $N \in \mathbb{N}$ such that $T_{n}(U) \cap V \neq \emptyset$ for all $n \geq N$. (See [13] for the case $\left\{T^{n}\right\}$.)

(5) $\left\{T_{n}\right\}$ is said to satisfy the Hypercyclicity Criterion for an increasing sequence $\left\{n_{k}\right\} \subset \mathbb{N}$ (cf. [8, Definition 1.1]) provided there exist dense subsets $X_{0}, Y_{0}$ of $X$ satisfying the following two conditions:

(a) for every $x \in X_{0}, T_{n_{k}} x \rightarrow 0$;

(b) for every $y \in Y_{0}$ there is a sequence $\left\{u_{k}\right\} \subset X$ such that $u_{k} \rightarrow 0$ and $T_{n_{k}} u_{k} \rightarrow y$.

Note that if an operator $T$ is hypercyclic (equivalently, topologically transitive) and $x$ is its hypercyclic vector, then each element of the dense set $\left\{T^{n} x\right\}_{n \in \mathbb{N}}$ is a hypercyclic vector, and hence $T$ is densely hypercyclic. More generally, Peris has shown (cf. [18, Proposition 1]) that if $\left\{T_{n}\right\}$ is a commuting sequence of operators with dense range, then $\left\{T_{n}\right\}$ is hypercyclic if and only if $\left\{T_{n}\right\}$ is densely hypercyclic.

K.-G. Grosse Erdmann [17, Satz 1.2.2] proved the following result.

Theorem 2.2. A sequence $\left\{T_{n}\right\} \subset L(X)$ is densely hypercyclic if and only if $\left\{T_{n}\right\}$ is topologically transitive.

Thus, for a commuting sequence $\left\{T_{n}\right\}$ of operators with dense range, hypercyclicity, dense hypercyclicity, and topological transitivity are equivalent notions. But a general hypercyclic sequence $\left\{T_{n}\right\}$ is not necessarily densely hypercyclic even when $\left\{T_{n}\right\}$ is hereditarily hypercyclic. For such an example we refer to [8, p. 23]. Thus topological transitivity of a general sequence of operators is stronger than its hypercyclicity.

Theorem 2.2 and the following theorem of Bernal-González and Grosse-Erdmann [8, Theorem 2.2] show that the Hypercyclicity Criterion is stronger than topological transitivity. 
Theorem 2.3. Let $\left\{T_{n}\right\} \subset L(X)$. Then the following are equivalent:

(i) $\left\{T_{n}\right\}$ satisfies the Hypercyclicity Criterion.

(ii) $\left\{T_{n}\right\}$ has a hereditarily densely hypercyclic subsequence.

(iii) for every $N \in \mathbb{N},\left(T_{n} \oplus \cdots \oplus T_{n}\right)_{N-\text { fold }}$ is densely hypercyclic on $X^{N}$.

Remark 2.4. Unlike the case of a single operator (cf. [23, Theorem 2.3]), for the sequence $\left\{T_{n}\right\}$ the Hypercyclicity Criterion is in general not equivalent to syndetical hypercyclicity. To give an example, choose a sequence $\left\{T_{n}^{(1)}\right\}$ which satisfies the Hypercyclicity Criterion and a nonhypercyclic sequence $\left\{T_{n}^{(2)}\right\}$, and combine them to define the sequence $\left\{T_{n}\right\}$ by $T_{2 k-1}=T_{k}^{(1)}$ and $T_{2 k}=T_{k}^{(2)}$ for $k \geq 1$. Then $\left\{T_{n}\right\}$ satisfies the Hypercyclicity Criterion. But, since $\left\{T_{2 n}\right\}$ is not hypercyclic, $\left\{T_{n}\right\}$ is not syndetically hypercyclic. Thus the Hypercyclicity Criterion does not imply syndetical hypercyclicity. The syndetical hypercyclicity does not imply the Hypercyclicity Criterion either. Indeed, L. Bernal and K.-G. Grosse-Erdmann [8, Remark $2.3(\mathrm{c})$ ] provided an example of a hereditarily hypercyclic sequence $\left\{T_{n}\right\}$ (so in particular, $\left\{T_{n}\right\}$ is syndetically hypercyclic) that does not satisfy the Hypercyclicity Criterion.

Next, we prove some necessary conditions for hypercyclicity, hereditary hypercyclicity, and topological mixing.

Proposition 2.5. Let $X$ be a Fréchet space and $\left\{T_{n}\right\} \subset L(X)$.

(i) If $\left\{T_{n}\right\}$ is hypercyclic, then for any $x \in X$ there exist a sequence $\left\{w_{k}\right\} \subset$ $H C\left(\left\{T_{n}\right\}\right.$ ) (dependent on $x$ ) and an increasing sequence $\left\{n_{k}\right\} \subset \mathbb{N}$ (dependent on $\left.\left\{w_{k}\right\}\right)$ such that $w_{k} \in B(0 ; 1 / k)$ and $T_{n_{k}} w_{k} \in B(x ; 1 / k)$ for all $k \in \mathbb{N}$.

(ii) If $\left\{T_{n}\right\}$ is almost-commuting and hypercyclic, then for any $z \in H C\left(\left\{T_{n}\right\}\right)$ there exists an increasing sequence $\left\{m_{k}\right\} \subset \mathbb{N}$ (dependent on $z$ ) such that $T_{m_{k}} x \rightarrow 0$ for all $x$ in the dense set $D_{z}:=\left\{T_{n} z\right\}_{n=1}^{\infty}$.

(iii) If $\left\{T_{n}\right\}$ is hereditarily hypercyclic, then for any $z \in X$ and any increasing sequence $\left\{m_{k}\right\} \subset \mathbb{N}$ there exist a sequence $\left\{w_{k}\right\} \subset H C\left(\left\{T_{m_{k}}\right\}\right)$ (dependent on $z$ and $\left\{m_{k}\right\}$ ) and a subsequence $\left\{n_{k}\right\}$ of $\left\{m_{k}\right\}$ (dependent on $\left\{w_{k}\right\}$ ) such that $w_{k} \in B(0 ; 1 / k)$ and $T_{n_{k}} w_{k} \in B(z ; 1 / k)$ for all $k \in \mathbb{N}$.

(iv) If $\left\{T_{n}\right\}$ is topologically mixing, then for any increasing sequence $\left\{m_{k}\right\} \subset \mathbb{N}$ $H C\left(\left\{T_{m_{k}}\right\}\right)$ is dense in $X$, and for any $z \in X$ there exist a subsequence $\left\{n_{k}\right\}$ of $\left\{m_{k}\right\}$ and a sequence $\left\{w_{k}\right\} \subset H C\left(\left\{T_{m_{k}}\right\}\right)$ (both dependent on $z$ and $\left\{m_{k}\right\}$ ) such that $w_{k} \in B(0 ; 1 / k)$ and $T_{n_{k}} w_{k} \in B(z ; 1 / k)$ for all $k \in \mathbb{N}$.

Proof. (i) Let $x_{0} \in H C\left(\left\{T_{n}\right\}\right)$ and $w_{k}:=\frac{x_{0}}{2 k d\left(x_{0}, 0\right)}$ for each $k \in \mathbb{N}$. Then $w_{k} \in$ $B(0 ; 1 / k) \cap H C\left(\left\{T_{n}\right\}\right)$. Thus $\left\{T_{n} w_{k}\right\}_{n=1}^{\infty}$ is dense in $X$, so that we can choose an increasing sequence $\left\{n_{k}\right\} \subset \mathbb{N}$ such that $T_{n_{k}} w_{k} \in B\left(x, \frac{1}{k}\right)$ for all $k=1,2, \ldots$

(ii) Since for $z \in H C\left(\left\{T_{n}\right\}\right) D_{z}:=\left\{T_{n} z\right\}_{n=1}^{\infty}$ is dense, there exists an increasing sequence $\left\{m_{k}\right\} \subset \mathbb{N}$ such that $T_{m_{k}} z \rightarrow 0$, and for each $x=T_{n} z \in D_{z}$ we have $\lim _{k \rightarrow \infty} T_{m_{k}} x=\lim _{k \rightarrow \infty} T_{m_{k}} T_{n} z=\lim _{k \rightarrow \infty} T_{n} T_{m_{k}} z=0$.

(iii) follows from (i).

(iv) If $\left\{T_{n}\right\}$ is topologically mixing, then $\left\{T_{n}\right\}$ must be hereditarily densely hypercyclic, by Theorem 2.6. Hence $H C\left(\left\{T_{m_{k}}\right\}\right)$ is dense in $X$ for any increasing sequence $\left\{m_{k}\right\} \subset \mathbb{N}$. The rest of the assertion follows from (iii).

The following is a known characterization of topological mixing. For completeness, we include here a short proof. A similar argument also appears in [16, Lemma $2.2]$ for the case of a sequence of the form $\left\{T_{n}\right\}=\left\{T^{n}\right\}$, where $T$ is a single operator. 
Theorem 2.6. For a sequence $\left\{T_{n}\right\} \subset L(X)$, the following conditions are equivalent:

(i) $\left\{T_{n}\right\}$ is topologically mixing.

(i') Every subsequence of $\left\{T_{n}\right\}$ is topologically mixing.

(ii) Every subsequence of $\left\{T_{n}\right\}$ is topologically transitive.

(iii) $\left\{T_{n}\right\}$ is hereditarily densely hypercyclic.

Proof. "(i) $\Rightarrow\left(\mathrm{i}^{\prime}\right) \Rightarrow$ (ii)" are obvious, and "(ii) $\Rightarrow$ (iii)" follows from Theorem 2.2.

(iii) $\Rightarrow$ (i). If $\left\{T_{n}\right\}$ is not topologically mixing, then there exists nonempty open sets $U, V$ and an increasing sequence $\left\{n_{k}\right\}$ such that $T_{n_{k}}(U) \cap V=\phi$ for each $k \in \mathbb{N}$. This implies that $U$ is disjoint from $H C\left(\left\{T_{n_{k}}\right\}\right)$, which means that $\left\{T_{n_{k}}\right\}$ is not densely hypercyclic, and so $\left\{T_{n}\right\}$ is not hereditarily densely hypercyclic.

Theorem 2.7. For a sequence $\left\{T_{n}\right\} \subset L(X)$, the following conditions have the relations: (i) $\Rightarrow$ (ii) $\Rightarrow$ (iii) + (iv).

(i) $\left\{T_{n}\right\}$ satisfies the Hypercyclicity Criterion with respect to the full sequence.

(ii) $\left\{T_{n}\right\}$ is topologically mixing.

(iii) $\left\{T_{n}\right\}$ satisfies the Hypercyclicity Criterion with respect to some increasing sequence $\left\{n_{k}\right\} \subset \mathbb{N}$.

(iv) $\left\{T_{n}\right\}$ satisfies condition (b) of the Hypercyclicity Criterion with respect to the full sequence, that is, there exists a dense subset $Y_{0}$ of $X$ such that for every $y \in Y_{0}$ there is a sequence $\left\{u_{n}\right\} \subset X$ such that $u_{n} \rightarrow 0$ and $T_{n} u_{n} \rightarrow y$.

Proof. (i) $\Rightarrow$ (ii). The argument below is similar to the one in J. Shapiro's book [24, p. 112], where the sequence $\left\{T^{n}\right\}$ is considered. Let $X_{0}, Y_{0}$ be the two dense sets in (5) of Definition 2.1 with $n_{k}=n$. Given any nonempty open sets $U, V$, there exist $x \in X_{0}, y \in Y_{0}, \varepsilon>0$ such that $B(x, \varepsilon) \subset U$ and $B(y, 2 \varepsilon) \subset V$. By the Hypercyclicity Criterion with respect to the full sequence, there is a large $N_{0} \in \mathbb{N}$ and $\left\{u_{n}\right\} \subset X$ such that the following hold for all $n>N_{0}: T_{n} x \in B(0, \varepsilon), u_{n} \in$ $B(0, \varepsilon)$, and $T_{n} u_{n}-y \in B(0, \varepsilon)$. Hence $x+u_{n} \in B(x, \varepsilon) \subset U$ and $T_{n}\left(x+u_{n}\right)=$ $y+T_{n} x+T_{n} u_{n}-y \in B(y, 2 \varepsilon) \subset V$ for all $n>N_{0}$. This shows $\left\{T_{n}\right\}$ is topologically mixing.

"(ii) $\Rightarrow$ (iii)" follows immediately from Theorem 2.6 and Theorem 2.3 (i.e., Theorem 2.2 in [8]).

(ii) $\Rightarrow$ (iv). For any $y \in Y_{0}:=X$, the assumption implies that there is an increasing sequence $\left\{n_{k}\right\} \subset \mathbb{N}$ such that for each $k \in \mathbb{N}$

$$
T_{n}\left(B\left(0, \frac{1}{k}\right)\right) \cap B\left(y, \frac{1}{k}\right) \neq \emptyset \text { for all } n \geq n_{k} .
$$

Thus for each $0 \leq l<n_{k+1}-n_{k}$, we can choose $u_{n_{k}+l} \in B\left(0, \frac{1}{k}\right)$ such that $T_{n_{k}+l} u_{n_{k}+l} \in B\left(y, \frac{1}{k}\right)$. Then $u_{n} \in B\left(0, \frac{1}{k}\right)$ and $T_{n} u_{n} \in B\left(y, \frac{1}{k}\right)$ for all $n_{k} \leq n<$ $n_{k+1}$. This shows that $u_{n} \rightarrow 0$ and $T_{n} u_{n} \rightarrow y$ as $n \rightarrow \infty$.

From Theorem 1.1 of [13] and Theorem 2.7 we can deduce the following corollary.

Corollary 2.8 ([5, Remark 3.3]). For an operator $T \in L(X)$, the sequence $\left\{T^{n}\right\}$ satisfies the Hypercyclicity Criterion with respect to the full sequence if and only if it satisfies the Hypercyclicity Criterion with respect to some syndetic sequence $\left\{n_{k}\right\}$.

Proof. The necessity is obvious. To show the sufficiency, suppose $\left\{T^{n}\right\}$ satisfies the Hypercyclicity Criterion with respect to some syndetic sequence $\left\{n_{k}\right\}$. By 
definition, there exists a dense subset $X_{0}$ such that $T^{n_{k}} x \rightarrow 0$ for all $x \in X_{0}$. Then we have

$$
\left\|T^{n} x\right\|=\left\|T^{n_{k}+r} x\right\| \leq \max _{0 \leq r \leq M}\|T\|^{r}\left\|T^{n_{k}} x\right\| \rightarrow 0 \text { as } n \rightarrow \infty,
$$

where $n=n_{k}+r$ for some $0 \leq r \leq M:=\sup _{k \in \mathbb{N}}\left\{n_{k+1}-n_{k}\right\}$. Hence $\left\{T^{n}\right\}$ satisfies condition (a) of the Hypercyclicity Criterion with respect to the full sequence.

On the other hand, since Theorem 1.1 of [13] asserts that $\left\{T^{n}\right\}$ is topologically mixing, by the assertion "(ii) $\Rightarrow$ (iv)" of Theorem $2.7,\left\{T^{n}\right\}$ also satisfies condition (b) of the Hypercyclicity Criterion with respect to the full sequence. Thus $\left\{T^{n}\right\}$ satisfies the Hypercyclicity Criterion with respect to the full sequence.

Remark 2.9. (1) Because of Corollary 2.8, the part "(i) $\Rightarrow$ (ii)" of Theorem 2.7 can be viewed as a generalization of Theorem 1.1 in 13 to sequences of operators. But, unlike the case of a single operator, for the sequence $\left\{T_{n}\right\}$ the Hypercyclicity Criterion with respect to a syndetic sequence does not imply being topologically

mixing. For, if in the example in Remark 2.4 we choose the sequence $\left\{T_{n}^{(1)}\right\}$ to satisfy the Hypercyclicity Criterion with respect to a syndetic sequence $\left\{n_{k}\right\}$, then the sequence $\left\{T_{n}\right\}$ constructed there satisfies the Hypercyclicity Criterion with respect to the syndetic sequence $\left\{n_{k}\right\}$. But, since $\left\{T_{2 n}\right\}$ is not hypercyclic, $\left\{T_{n}\right\}$ is not topologically mixing, by Theorem 2.6.

(2) While the assertion "(ii) $\Rightarrow$ (iii) + (iv)" in Theorem 2.7 holds, there exist sequences $\left\{T_{n}\right\}=\left\{T^{n}\right\}$ (e.g., when $T=I+K$ is the perturbation of the identity by the compact unilateral backward shift $K:\left(x_{0}, x_{1}, x_{3}, \ldots\right) \mapsto\left(2^{-1} x_{1}, 2^{-2} x_{2}, 2^{-3} x_{3}, \ldots\right)$ acting on $X=l_{2}$ ) that are topologically mixing and do not satisfy condition (a) of the Hypercyclicity Criterion with respect to any syndetic sequence $\left\{n_{k}\right\}$; see [16. Theorem 2.5] and [5, Remark 3.3]. Hence, a topologically mixing operator $T$, though, must satisfy the Hypercyclicity Criterion with respect to some increasing sequence $\left\{n_{k}\right\} \subset \mathbb{N}$, and may not satisfy the Hypercyclicity Criterion with respect to any syndetic sequence, i.e., the answer to the question raised by Costakis and Sambarino [13, P. 386] is "No".

\section{EXAMPLES}

Consider the composition operators on the spaces of holomorphic function $H(G)$ for some $G \subset \mathbb{C}$ given by

$$
T_{n} f=f \circ \varphi_{n}, \quad f \in H(G),
$$

where $\varphi_{n}: G \rightarrow G, n \in \mathbb{N}$, are automorphisms on $G$.

Bernal and Montes ([9, 22]) proved that if $G$ is not conformally equivalent to $\mathbb{C} \backslash\{0\}$, then $\left\{T_{n}\right\}$ is hypercyclic if and only if $\left\{\varphi_{n}\right\}$ is a run-away sequence, i.e. for every compact subset $K \subset G$ there exists some $n \in \mathbb{N}$ with $K \cap \varphi_{n}(K)=\emptyset$. Recently, the following proposition was proved in [8, Proposition 2.4].

Proposition 3.1. Let $\left\{T_{n}\right\}$ be a sequence of composition operators as above. Then the following assertions are equivalent:

(i) $\left\{T_{n}\right\}$ is hypercyclic;

(ii) $\left\{T_{n}\right\}$ has a hereditarily densely hypercyclic subsequence;

(iii) $\left\{T_{n}\right\}$ satisfies the Hypercyclicity Criterion;

(iv) $\left\{\varphi_{n}\right\}$ is a run-away sequence. 
From this and Theorem 2.6 one can deduce the following theorem which characterizes $\left\{T_{n}\right\}$ being topologically mixing by $\left\{\phi_{n}\right\}$ being strongly running away.

Theorem 3.2. Let $\left\{T_{n}\right\}$ be a sequence of composition operators $T_{n} f=f \circ \varphi_{n}$ on a nonempty open subset $G$ of $\mathbb{C}$ that is not conformally equivalent to $\mathbb{C} \backslash\{0\}$. Then the following assertions are equivalent.

(i) $\left\{T_{n}\right\}$ is topologically mixing;

(ii) $\left\{T_{n}\right\}$ is hereditarily densely hypercyclic;

(iii) $\left\{\varphi_{n}\right\}$ is a strongly run-away sequence, i.e. for every compact subset $K \subset G$ there exists some $N \in \mathbb{N}$ with $K \cap \varphi_{n}(K)=\emptyset$ for all $n \geq N$.

Proof. The equivalence of (i) and (ii) have been proved in Theorem 2.6.

(i) $\Rightarrow$ (iii). By Theorem 2.6 we know that $\left\{T_{n}\right\}$ is hereditarily densely hypercyclic with the full sequence. Suppose (iii) is false. Then there exists a compact subset $K^{\prime} \subset G$ and a sequence $\left\{n_{k}\right\} \subset \mathbb{N}$ such that $K^{\prime} \cap \varphi_{n_{k}}\left(K^{\prime}\right) \neq \emptyset$ for all $k \geq 1$, i.e., $\left\{\varphi_{n_{k}}\right\}$ is not a run-away sequence. By Proposition 3.1, $\left\{T_{n_{k}}\right\}$ is not hypercyclic and hence not densely hypercyclic. This is a contradiction.

(iii) $\Rightarrow$ (ii). Since (iii) implies that every subsequence $\left\{\phi_{n_{k}}\right\}$ is run-away, $\left\{T_{n_{k}}\right\}$ is hypercyclic and thus is densely hypercyclic, by Proposition 3.1. Hence $\left\{T_{n}\right\}$ is hereditarily densely hypercyclic.

Let $H(\mathbb{C})$ be the entire function over $\mathbb{C}$ endowed with the compact-open topology, for which a sequence $\left\{f_{n}\right\} \subset H(\mathbb{C})$ converges to 0 means that $\lim _{n \rightarrow \infty} \sup _{|z| \leq R}\left|f_{n}(z)\right|=0$ for all $R>0$. That is, $f_{n} \rightarrow 0$ if and only if for all $R>0$ and all $\varepsilon>0$, there exists an $N$ such that for all $n>N$,

$$
\left|f_{n}(z)\right|<\varepsilon \text { for all }|z| \leq R \text {. }
$$

In [3, it was shown that the "weighted differentiation" operator $T_{\lambda}, T_{\lambda}(f)(z)=$ $f^{\prime}(\lambda z)$ acting on $H(\mathbb{C})$ is hypercyclic if and only if $|\lambda| \geq 1$. It can also be observed from the proof of Theorem 13 in 3 that $T_{\lambda}$ is hypercyclic if and only if it satisfies the Hypercyclicity Criterion with respect to the full sequence. In the following theorem, we consider topological mixing of a sequence $\left\{T_{n}\right\}$ of weighted differential operators defined below.

Theorem 3.3. For a bounded sequence $\left\{a_{n}\right\} \subset \mathbb{C}$ with 0 not belonging to $\overline{\left\{a_{n}\right\}}$ and $\left\{b_{n}\right\} \subset \mathbb{C}$, define $T_{n}: H(\mathbb{C}) \rightarrow H(\mathbb{C})$ by

$$
T_{n}(f)(z)=b_{n}^{n} f^{(n)}\left(a_{n} z\right) .
$$

Then the following are equivalent:

(i) $\left|b_{n}\right|^{-n}=o\left(\frac{n^{n+\frac{1}{2}}}{(\operatorname{Re})^{n}}\right)$ for all $R>0$;

(ii) $\left\{T_{n}\right\}$ satisfies the Hypercyclicity Criterion with respect to the full sequence;

(iii) $\left\{T_{n}\right\}$ is topologically mixing.

Proof. (i) $\Rightarrow$ (ii). Let $X_{0}=Y_{0}$ be the set of all polynomials, which is dense in $H(\mathbb{C})$. For any $p(z)=\sum_{k=0}^{m} c_{k} z^{k} \in X_{0}$, we obviously have $T_{n} p=0$ for all $n>m$, showing condition (a) of (5) in Definition 2.1. On the other hand, let $u_{n}(z)=\sum_{k=0}^{m} \frac{c_{k}}{b_{n}^{n}} \frac{k ! z^{k+n}}{(k+n) ! a_{n}^{k}}$. It is easy to check that $T_{n} u_{n}=p$. Using Stirling's 
formula, $n ! \sim n^{n+\frac{1}{2}} e^{-n}$ as $n \rightarrow \infty$, we have for $R>0$

$$
\begin{aligned}
\max _{|z| \leq R}\left|u_{n}(z)\right| & \leq \sum_{k=0}^{m}\left|c_{k}\right| \max _{|z| \leq R} \frac{k !|z|^{k+n}}{(k+n) !\left|a_{n}\right|^{k}\left|b_{n}\right|^{n}} \\
& \leq \sum_{k=0}^{m}\left|c_{k}\right| \frac{k !|R|^{k+n}}{(k+n) !\left|a_{n}\right|^{k}\left|b_{n}\right|^{n}} \\
& \sim \sum_{k=0}^{m}\left|c_{k}\right| \frac{k !|R|^{k+n} e^{n+k}}{(k+n)^{n+k+\frac{1}{2}}\left|a_{n}\right|^{k}\left|b_{n}\right|^{n}} \\
& \leq K \frac{|R|^{n} e^{n}}{(n)^{n+\frac{1}{2}}\left|b_{n}\right|^{n}} \quad \text { for some } K>0 .
\end{aligned}
$$

Since the assumption implies that the last term tends to 0 as $n \rightarrow \infty$, we have $u_{n} \rightarrow 0$ in $H(\mathbb{C})$. This shows (b) of (5) in Definition 2.1.

(ii) $\Rightarrow$ (iii). This is proved in Theorem 2.7.

(iii) $\Rightarrow$ (i). Suppose to the contrary, there exist $\epsilon, R>0$ and an increasing sequence $\left\{n_{k}\right\} \subset \mathbb{N}$ such that $\frac{|R|^{n_{k}} e^{n_{k}}}{\left(n_{k}\right)^{n_{k}+\frac{1}{2}}\left|b_{n_{k}}\right|^{n_{k}}} \geq \epsilon$ for all $k \geq 1$. Therefore, by Cauchy's inequality and Stirling's formula we infer that for every $f \in H(\mathbb{C})$,

$$
\begin{aligned}
\max _{|z| \leq R}\left|T_{n_{k}}(f)(z)\right| & =\max _{|z| \leq R}\left|b_{n_{k}}^{n_{k}} f^{\left(n_{k}\right)}\left(a_{n_{k}} z\right)\right| \\
& \leq\left|b_{n_{k}}\right|^{n_{k}} \max _{|z| \leq R}\left\{\frac{n_{k} ! \max _{\left|z^{\prime}-a_{n_{k}} z\right| \leq r}\left|f\left(z^{\prime}\right)\right|}{r^{n_{k}}}\right\} \text { for any } r>0 \\
& \leq \frac{M_{r}\left|b_{n_{k}}\right|^{n_{k}} n_{k} !}{r^{n_{k}}} \text { for some } M_{r}>0 \\
& \leq \frac{K M_{r}\left|b_{n_{k}}\right|^{n_{k}}\left(n_{k}\right)^{n_{k}+\frac{1}{2}}}{r^{n_{k}} e^{n_{k}}} \text { for some } K, M_{r}>0 .
\end{aligned}
$$

If we let $r=R$, then $\max _{|z| \leq R}\left|T_{n_{k}}(f)(z)\right| \leq K M_{R} / \epsilon$ for all $k \geq 1$, so that no subsequence of $\left\{T_{n_{k}} f\right\}$ converges to the constant function $K M_{R} / \epsilon+1$. Thus $\left\{T_{n_{k}}\right\}$ is not hypercyclic, and hence $\left\{T_{n}\right\}$ is not topologically mixing by Theorem 2.6.

\section{ACKNOWLEDGEMENT}

The authors are grateful to the referee for informing them of several recent papers and for valuable suggestions which resulted in the correction of a mistake and the improvement of the presentation of this paper.

\section{REFERENCES}

[1] S. I. Ansari, Hypercyclic and cyclic vectors, J. Funct. Anal. 128 (1995), 374-383. MR1319961 (96h:47002)

[2] S. I. Ansari, Existence of hypercyclic operators on topological vector spaces, J. Funct. Anal. 148 (1997), no. 2, 384-390. MR1469346 (98h:47028a)

[3] R. Aron and D. Markose, On universal functions, J. Korean Math. Soc. 41 (2004), 65-76. MR 2048701 (2005b:30029)

[4] J. Banks, Topological mapping properties defined by digraphs, Discrete and Cont. Dyn. Syst. 5 (1999), 83-92. MR:1664461 (99j:54038) 
[5] T. Bermúdez, A. Bonilla, J.A. Conejero and A. Peris, Hypercyclic, topologically mixing and chaotic semigroups on Banach spaces, Studia Math. 170 (2005), no. 1, 57-75. MR2142183

[6] L. Bernal-González, On hypercyclic operators on Banach spaces, Proc. Amer. Math. Soc. 127 (1999), no. 4, 1003-1010. MR.1476119 (99f:47010)

[7] L. Bernal-González, Densely hereditarily hypercyclic sequences and large hypercyclic manifolds, Proc. Amer. Math. Soc. 127 (1999), 3279-3285. MR1646318 (2000b:47047)

[8] L. Bernal-González and K.-G. Grosse-Erdmann, The Hypercyclicity Criterion for sequences of operators, Studia Math. 157 (2003), 17-32. MR.1980114 (2003m:47013)

[9] L. Bernal-González and A. Montes-Rodríguez, Universal functions for composition operators, Complex Variables Theory Appl. 27 (1995), 47-56. MR1316270(96a:30041)

[10] J. Bès and A. Peris, Hereditarily hypercyclic operators, J. Funct. Anal. 167 (1999), 94-112. MR:1710637 (2000f:47012)

[11] G.D. Birkhoff, Démonstration dún théoréme elémentaire sur les fonctions entiéres, $C . R$. Acad. Sci. Paris 189 (1929), 473-475.

[12] J. Bonet and A. Peris, Hypercyclicity operators on non-normable Fréchet spaces, J. Funct. Anal. 159 (1998), no. 2, 587-595. MR1658096 (99k:47044)

[13] G. Costakis and M. Sambarino, Topologically mixing hypercyclic operators, Proc. Amer. Math. Soc. 132 (2003), 385-389. MR2022360 (2004i:47017)

[14] R. M. Gethner and J. H. Shapiro, Universal vectors for operators on space of holomorphic functions, Proc. Amer. Math. Soc. 100 (1987), 281-288. MR0884467 (88g:47060)

[15] G. Godefroy and J. H. Shapiro, Operators with dense, invariant, cyclic vector manifolds, J. Funct. Anal. 98 (1991), no. 2, 229-269. MR.1111569 (92d:47029)

[16] S. Grivaux, Some observations regarding the Hypercyclicity Criterion, preprint.

[17] K.-G. Grosse-Erdmann, Holomorphe Monster und Universelle Funktionen, Mitt. Math. Sem. Giessen. 176 (1987). MR0877464 (88i:30060)

[18] K.-G. Grosse-Erdmann, Universal families and hypercyclic operators, Bull. Amer. Math Soc. 36 (1999), 345-381. MR.1685272 (2000c:47001)

[19] C. Kitai, Invariant Closed Sets for Linear Operators, Ph.D. thesis, University of Toronto (1982).

[20] F. León-Saavedra and V. Müller, Hypercyclic sequences of operators, Studia Math., to appear.

[21] G. MacLane, Sequences of derivatives and normal families, J. Analyse Math. 2 (1952), 72-87. MR0053231 (14:741d)

[22] A. Montes-Rodríguez, A note on Birkhoff open sets, Complex Variables Theory Appl. 30 (1996), 193-198. MR1404989 (97h:30056)

[23] A. Peris and L. Saldivia, Syndetically hypercyclic operators, Integr. Equ. Oper. Theory, 51 (2005), 275-281. MR2120081 (2005h:47017)

[24] J.H. Shapiro, Composition Operators and Classical Function Theory, Springer-Verlag, NY (1993). MR1237406 (94k:47049)

Department of Mathematics, National Central University, Chung-Li, 320 Taiwan

Graduate School of Engineering, Lunghwa University of Science and Technology, Gueishan, TaOyuan, 333 Taiwan

E-mail address: shaw@math.ncu.edu.tw 